\title{
Efficacy of Autologous Cultured Fibroblast Cells as a Treatment for Patients with Facial Contour Defects: A Clinical Replication Study
}

\author{
Edward Charles R. Lee", Kim Siea Lee², Jezamine Lim³ ${ }^{3}$ Jason Yip ${ }^{4}$, Cheng Zhang5, Xu Zhang5, \\ Han Bin ${ }^{5}$, To Lim6* \\ ${ }^{1}$ Sabah Medical Centre, Sabah, Malaysia \\ ${ }^{2}$ Loh Guan Lye Specialists Centre, Pinang, Malaysia \\ ${ }^{3}$ Tissue Engineering Centre, University Kebangsaan, Kuala Lumpur, Malaysia \\ ${ }^{4}$ Astute Clinic, Kuala Lumpur, Malaysia \\ ${ }^{5}$ Beijing Cell Technology Inc., Beijing, China \\ ${ }^{6}$ SB Clinical Research, Malaysia \\ Email: *limteckonn@gmail.com
}

How to cite this paper: Lee, E.C.R., Lee, K.S., Lim, J., Yip, J., Zhang, C., Zhang, X., Bin, H. and Lim, T. (2017) Efficacy of Autologous Cultured Fibroblast Cells as a Treatment for Patients with Facial Contour Defects: A Clinical Replication Study. Journal of Cosmetics, Dermatological Sciences and Applications, 7, 306-317. https://doi.org/10.4236/jcdsa.2017.74027

Received: December 24, 2013

Accepted: November 17, 2017

Published: November 20, 2017

Copyright ( 2017 by authors and Scientific Research Publishing Inc. This work is licensed under the Creative Commons Attribution International License (CC BY 4.0).

http://creativecommons.org/licenses/by/4.0/ (c) (i) Open Access

\begin{abstract}
Background: The US FDA has recently approved autologous cultured fibroblast cells (ACF) as treatment for cutaneous contour defects. ACF provides an alternative to synthetic fillers or fat grafting with the significant advantage of producing longer lasting effect. Methods: This was a prospective open label single group clinical study to demonstrate the clinical efficacy of ACF that we have replicated in our lab. The study enrolled 18 patients with nasolabial folds from 2 centres in Malaysia in 2011-2012. ACF at dose of 23 or 92 million cells were injected on 3 occasions at 4 weeks interval. Efficacy at 6- and 12-month post treatment was assessed using a standardized 7-point scale which was performed by the 2 investigators as well as a panel of 7 independent evaluators. Results: We obtained a successful outcome (primary endpoint), defined as improvement in the appearance of the nasolabial fold of at least 2 points on the 7 -point scale, in $33 \%$ of patients as assessed by investigators at 12 -month follow-up and in $22 \%$ of patients assessed by independent evaluators. Investigators also found $78 \%$ of patients having had a 1-point improvement from baseline while independent evaluators found $83 \%$ of patients having improved by as much. Patients were uniformly (100\%) satisfied with the improvement in their appearance. No treatment-related adverse event was reported. Baseline score and follow-up duration had significant effect on treatment response. The greater the baseline severity and the longer the post-treatment follow-up, the better the response to ACF. Larger dose of cells (92 million cells) has little additional effect suggesting that the $1 \mathrm{~mL}$ dose $(23$ million
\end{abstract}


cells) is near optimal. Older patients showed a trend towards poorer response but this was not statistically significant. Conclusions: We have successfully replicated the laboratory method and clinical procedure to perform ACF treatment which was effective in improving the appearance of nasolabial folds in some patients.

\section{Keywords}

Facial Contour Defect, Nasolabial Fold, Wrinkle, Autologous Cultured Fibroblast Cell, Autologous Cell Therapy

\section{Introduction}

Cutaneous contour defects consist of skin soft tissue abnormalities such as superficial wrinkles around the eyes, lip, deep fold or furrows in the forehead, glabella, nasolabial and peri-oral region, or scarring of the skin due to acne, chicken pox, trauma or burns. Many patients seek correction for these defects especially those affecting the face.

Available treatments for these defects include dermal fillers and fat grating. Filler has been around for more than 30 years and still has an important role [1]. Major advantages with filler are that it requires only a simple procedure to administer at point of care and achieve an immediately obvious volume-filling effect. However, it carries a risk of hyper-sensitivity reaction even with the use of modern synthetic filler such as Hyaluronic acid. Its effect is also temporary lasting for at most 12 months, and thus would require repeated treatments to maintain the correction. Autologous fat grafting, often in combination with stromal vascular fraction, is another popular treatment for contour defects [2] [3]. It uses simple procedures to harvest fat, to isolate the SVF and subsequently to inject into the recipient site; the entire process could be completed at the point of care. For the small volume of fat required to correct facial contour defects, it is easily accomplished even for thin persons with little body fat. However the evidence base supporting its efficacy remains questionable; indeed its most significant problem is unpredictable volume loss post-grafting with resorption rates ranging from $25 \%$ to $80 \%$ [3]-[8]. Graft survival could be significantly improved with addition of ex-vivo expanded adipose derived stem cells (ASC) [9], but that makes the procedure more complex.

More recently, the US FDA has approved a new treatment for cutaneous contour defects, the autologous cultured fibroblast cells (ACF).Histopathology studies on human volunteers have demonstrated that the injected human fibroblast cells were incorporated into the dermal structure and began production of collagen or stimulated synthesis from the native cells creating the thicker collagen layer [10] [11]. Subsequent clinical studies [12] including a randomized placebo controlled trial [13] have confirmed its clinical efficacy and safety in human. $\mathrm{ACF}$, like filler, requires only a simple intradermal injection procedure to admi- 
nister but it has no risk of hypersensitivity reaction. Like ASC enriched fat grafting, it requires specialized lab facility to perform the cell culture ex vivo, which clearly cannot be provided at point of care. Cell culture also carries the risk of contamination and risk of disease transmission from reagents derived from xenogeneic sources [14]. In addition, cell culture could potentially change the cells' phenotype under culture conditions [15]. The most significant advantage of ACF is its long-lasting effect and that sustained improvements up to 48 months have been observed to date [10].

We have replicated the fibroblast cell culture technique in our lab. This report presents the results of a clinical replication study to demonstrate the clinical efficacy and safety of the cell therapy as performed by our lab and clinic. This is to ensure that we could replicate the same good results as those reported by others in the literature [12] [13].

\section{Methods}

We conducted a prospective open label single group clinical trial at two centres in Malaysia to replicate the clinical efficacy of autologous cultured fibroblast cell (ACF) therapy. The study was conducted in accordance with Declaration of Helsinki and Good Clinical Practice (GCP) requirements. The institutional review-board of the study sites approved the protocol, and all patients gave written informed consent.

\subsection{Study Patients}

The inclusion criteria were age between 18 and 75 years, patients who were "dissatisfied" or "very dissatisfied" with the appearance of their nasolabial fold (score 4 or higher on a 5-point satisfaction scale) and the severity of which as assessed by the investigator were "moderate" or worse (Grade 4 or higher on a standardized 7-point ordinal severity scale with a photoguide).

The exclusion criteria were pregnant or nursing woman; evidence of HIV, hepatitis B and C, active systemic infection, active or chronic skin disease (such as psoriasis, eczema, blistering skin disease or local infection), severe keloid at BCG vaccination site), on chronic antibiotic or steroid therapy; history of organ transplant, autoimmune disease, alcohol or substance abuse, cancer.

\subsection{Cell Cultures}

Autologous fibroblast cells were isolated from a skin biopsy specimen (epidermal and dermal layers, $3 \times 7 \mathrm{~mm}^{2}$ ) taken from the patient's skin behind the ear and was transported to the lab in cryo vial filled with $2 \mathrm{~mL}$ culture media. Thermo Scientific Hyclone classical liquid media Dulbecco's Modified Eagles Medium, High Glucose (DMEM/High): with $4500 \mathrm{mg} / \mathrm{L}$ Glucose, with $4.0 \mathrm{~mm}$ L-Glutamine, without Sodium Pyruvate was used throughout the cell culture process. The skin biopsy was washed $4-6$ times with culture media and excess fat was removed by using forceps and scissors. It was then transferred to a 60 
$\mathrm{mm}$ petri dish, minced into small pieces and scattered over the petri dish before leaving it to dry for 10mins. Meanwhile, microbial screening for bacteria, mould and yeast was performed on the culture medium which was used to transport the biopsy tissue by spreading sufficient amount of medium onto nutrient agar and potato dextrose agar plates accordingly and observed after 3 - 5 days incubation at $37^{\circ} \mathrm{C}$. Once the biopsy tissue had adhered onto the petri dish, $3 \mathrm{~mL}$ culture medium mixture (DMEM/High, 10\% characterized foetal bovine serum and 1\% penicillin-streptomycin, all from Thermo Scientific Hyclone ${ }^{\oplus}$ ) was added gently into the petri dish and incubated at $36.8^{\circ} \mathrm{C}$ with $5 \% \mathrm{CO}_{2}$. Medium was changed every 2 - 3 days and fibroblast cell growth was monitored under phase contrast microscope.

Subculture was performed when primary culture reached $90 \%$ confluence, cell concentration was determined to be at $2.1-2.4 \times 10^{7}$ cells $/ \mathrm{mL}$ on average through cell count. Fibroblast cells were washed with DMEM/High and $1 \mathrm{~mL}$ $0.25 \%$ trypsin-EDTA was added into the petri dish for 5 min incubation at $37^{\circ} \mathrm{C}$ with $5 \% \mathrm{CO}_{2}$. Skin tissue might be detached from the petri dish under prolonged trypsinization process, hence $3 \mathrm{~mL}$ of culture medium mixture was added next to inactivate trypsin and cell suspension fluid was centrifuged at $1000 \mathrm{rpm}$ for 5 min to separate the fibroblast cells from supernatant. Once the cells suspension fluid was transferred to the centrifuge tube, $3 \mathrm{~mL}$ culture media mixture was added immediately to the primary culture dish to maintain fibroblast cells growth. The supernatant was then aspirated and $7 \mathrm{~mL}$ culture medium mixture with additional epidermal growth factor (EGF) was added into the centrifuge tube to re-suspend fibroblast cells and transferred to a $100 \mathrm{~mm}$ petri dish and culture was continued in a $5 \% \mathrm{CO}_{2}$ incubator at $37^{\circ} \mathrm{C}$. From first passage onwards, culture medium mixture with extra EGF was used to promote cell growth and temperature of $\mathrm{CO}_{2}$ incubator was maintained at $37^{\circ} \mathrm{C}$ as well.

Fibroblast cells were harvested when the amount of cells were sufficient for treatment purpose, which is usually between the fourth and sixth passage. Microbial screening was carried out during the last media change which was 3 days before cell harvesting to ensure sterility of the culture. One day prior to cell harvesting, the media in all petri dishes were changed to DMEM/High, thus the culture was free from serum and antibiotics. On the harvest day, culture media was aspirated and trypsinization process was performed as described previously. After centrifugation at $1000 \mathrm{rpm}$ for $5 \mathrm{~min}$, the supernatant was discarded and $10 \mathrm{~mL}$ DMEM/High was added to the fibroblast cells pellet and mixed gently with a serological pipette followed by centrifugation again at $1000 \mathrm{rpm}$ for another $5 \mathrm{~min}$. The washing procedure was repeated again with DMEM/High and then washed twice using multiple electrolytes injection fluid. During the final centrifugation, supernatant was aspirated and fibroblast cells pellet were transferred into a $2 \mathrm{~mL}$ cryo vial before sending to the designated clinical centre where the ACF treatment is to be administered. Endotoxin test using Limulus ES-II (Wako, Japan) was performed on the same day prior to injection.

Fibroblast cells for each patient were preserved for future use after all 3 injec- 
tions were completed. Procedures for cryopreserve were similar to cell harvesting. Five cryo vials with $1 \mathrm{~mL}$ fibroblast cells in each were prepared and kept at $2^{\circ} \mathrm{C}-8^{\circ} \mathrm{C}$ for $30 \mathrm{~min},-20^{\circ} \mathrm{C}$ for $30 \mathrm{~min}$ and overnight at $-80^{\circ} \mathrm{C}$ accordingly before moving to liquid nitrogen cryo tank for cell preservation.

\subsection{Cultured Fibroblast Cells Treatment}

The suspension of cultured fibroblast cells in cryo-vial was delivered to the treating physician for intradermal injection into the patient's nasolabial fold. One $\mathrm{ml}$ of the cell suspension contains about 23 million fibroblast cells (range 21 to 24 million cells per $\mathrm{mL}$ ). Each patient received a course of therapy consisting of three injections administered at an interval of 5 weeks. Theoretically number of $\mathrm{mL}$ (cell dosing) administered per injection varied according to length of the nasolabial fold to be treated. For this trial, 12 patients were treated with $1 \mathrm{~mL}$ per injection ( $0.5 \mathrm{~mL}$ for each side of the bilateral nasolabial folds) and another $7 \mathrm{pa}-$ tients were treated with $4 \mathrm{~mL}$ per injection.

\subsection{Study Assessments and Endpoints}

Study visits post-injection occurred at the second week, then monthly till to month 6 and one final visit at month 12 for efficacy and safety assessments.

The primary efficacy endpoint consists of 7 independent evaluator's assessment of the photographs of the bilateral nasolabial folds taken before treatment, at 6- and 12-month after completing treatment, on a standardized 7-point ordinal severity scale with a photoguide. An efficacious response is defined as a 2-point improvement from the baseline (pre-treatment) score on the 7-point scale in at least one treated area. The evaluators were not involved in the patients' care, nor did they participate in any laboratory or clinical procedure.

Other efficacy endpoints are:

- Investigator's live assessment of the change in facial contour defects before and after treatment using the same 7-point photoguide;

- Subject's live assessment of their satisfaction with the change in facial contour defects before and after treatment using a 5-point scale;

- Histological features of the healthy skin biopsy specimen compared with the features of an injected post-auricular site taken from a repeat biopsy 6-month after the injection. However, no patients consented to repeat biopsy.

\subsection{Statistical Methods}

Sample size was estimated based on the primary efficacy parameter which was the percent of patients who had an efficacious response to treatment. We assume this would be 75\% [10] [12] [13], and we would have no interest in ACF if we were unable to achieve at least $45 \%$ response rate. By using the Simon's minimax design [16] with type 1 and 2 errors of 0.1 , a total of 19 patients would be enrolled and ACF would be rejected as ineffective if we were to observe a response rate of less than $42 \%$ (11 or more non-response). 
Continuous variables were described by summary statistics such as mean, median, and standard deviation and categorical (nominal/ordinal) variables, by the frequencies of each category.

Ordinal regression (Proportional odds model [17] [18]) was used to estimate the effects of covariates on the response to ACF therapy as measured by the change from baseline in the independent evaluators' assessment of the severity of the patients' nasolabial fold on the 7-point scale. The model includes patients' age, body weight, baseline severity, duration after treatment (6 and 12 months) and dose ( $1 \mathrm{~mL}$ and $4 \mathrm{~mL})$.

In proportional odds model, the cumulative probabilities for the ordinal dependent variable, after suitable transformation (log it transform), is modelled as a linear function of covariates. The regression coefficient has interpretation as cumulative odds ratio (OR) [19]. We explain this by an example. In Table 3, the cumulative OR for the response to treatment at 12-month post-treatment compare to 6-month is 3.2. This means the odds for a better response to treatment are 3.2 times higher at 12-month after treatment than at 6-month. In other words, the cumulative distribution for the response to treatment at 12-month post treatment is shifted to the right of the distribution at 6-month follow-up. A key assumption for the model is the proportional odds assumption, that is, homogeneity of cut-point specific odds ratio. We assessed this informally by plotting the binary logistic odds ratio and its 95\% confidence interval against the cut-points [19]. No obvious heterogeneity in cut-point specific odds ratio was apparent.

The level of significance was set at 0.05 . To account for cluster sampling (each patients were scored multiple times by the 7 independent evaluators), we obtained robust variance estimates using the Huber's [20] or sandwich estimator.

\section{Results}

We enrolled a total of 19 patients for this study. One patient was mistakenly enrolled and treated for acne scar instead of nasolabial fold. She is excluded from analysis. All other patients had completed 6-month follow-up and are included in this analysis. All but 2 patients completed 12-month follow-up.

\subsection{Baseline features}

Baseline characteristics of these patients are shown in Table 1.

The mean (SD) age of the patients was 54 years (range 26 to 70 ), and $94 \%$ of them were women. No patients had previous treatment for their nasolabial folds.

\subsection{ACF injection}

Eleven patients were treated with a dose of $1 \mathrm{~mL}$ per injection $(0.5 \mathrm{~mL}$ each side of the nasolabial fold) while 7 patients were given a higher dose of $4 \mathrm{~mL}$ per injection ( $2 \mathrm{~mL}$ each side of the nasolabial fold). No local or systemic complications were reported. 
Table 1. Demographic and other baseline characteristics of subjects enrolled in the trial.

\begin{tabular}{cc}
\hline Baseline characteristics & N = 18 \\
\hline Mean age (SD), years & $54(9)$ \\
No. (\%) male patients & $1(5 \%)$ \\
Mean body weight (kg) & $55.5(7.5)$ \\
No. (\%) non-smoker & $18(100)$ \\
No. (\%) without previous treatment for nasolabial fold & $18(100)$ \\
\hline
\end{tabular}

No patients had any post-injection adverse events (AE). AE that were specifically sought included local pain, inflammation, bleeding and systemic reaction.

\subsection{Outcomes}

The outcome of ACF treatment is summarized in Table 2.

All 18 patients (100\%) completed the study and were included in the analysis.

For the primary endpoint, only 4 patients (22\%) had improved by 2 or more points on the 7 -point scale, short of the required $45 \%$ response rate. However, fifteen patients $(83 \%)$ had at least 1 point improvement on the 7-point scale at 12-month follow-up. All patients (100\%) by self-assessment were satisfied with the improvement in the appearance of their nasolabial folds. Figure 1 shows the typical improvement observed at 12-month follow-up in the nasolabial fold of a patient who had 3 injections of ACF 4 weeks apart.

Table 3 shows the effect of patients' age, body weight, baseline severity, duration after treatment ( 6 and 12 months) and cell dose $(1 \mathrm{~mL}$ and $4 \mathrm{~mL}$ ) on the response to ACF treatment. Baseline score and follow-up duration had significantly marked effect on response; the greater the baseline severity and the longer the post-treatment follow-up, the better the response to ACF. Interestingly a much larger dose of cells (92 million cells) has little additional effect than the 1 $\mathrm{mL}$ dose ( 23 million cells). Higher age ( $>60$ years) showed a trend towards poorer response but this was not statistically significant.

\section{Discussion}

In this prospective study, we have administered autologous cultured fibroblast cells (ACF) to 18 patients for the correction of bilateral nasolabial folds. We obtained an improvement in the appearance of the nasolabial fold of at least 1 point on a 7 -point scale, in about $80 \%$ of patients at 12 -month follow-up as evaluated by both independent evaluators and the investigators. Investigators were more optimistic in their assessment in finding $33 \%$ of patients having had a 2-point improvement from baseline while independent evaluators found only $22 \%$ of patients having improved by as much. Patients were uniformly (100\%) satisfied with the improvement in the appearance of their nasolabial folds. Our results therefore indicate that the regenerated dermis from the ACF treatment had successfully corrected the facial contour defects at 1 year after treatment.

Our results however did not compare favourably to the original ACF [13], which has recently received marketing authorization from the US FDA [21] and 
Table 2. Outcomes of fibroblast treatment among 18 patients.

\begin{tabular}{|c|c|c|}
\hline Outcome & Time on trial & $\begin{array}{l}\text { Results } \\
\mathrm{N}=18\end{array}$ \\
\hline $\begin{array}{c}\text { Independent evaluator's photograph } \\
\text { assessment of severity on a } \\
\text { 7-point ordinal scale } \\
\text { Primary End-point }\end{array}$ & & \\
\hline \multirow{3}{*}{$\begin{array}{l}\text { Primary End-point } \\
\text { No. (\%) with at least 2-point improvement from } \\
\text { baseline score }\end{array}$} & Baseline & $0(0)$ \\
\hline & 6-month post treatment & $1(6)$ \\
\hline & 12 -month post treatment & $4(22)$ \\
\hline \multirow[t]{3}{*}{$\begin{array}{l}\text { No. (\%) with at least 1-point improvement from } \\
\text { baseline score }\end{array}$} & Baseline & $0(0)$ \\
\hline & 6-month post treatment & $8(44)$ \\
\hline & 12-month post treatment & $15(83)$ \\
\hline \multirow{3}{*}{$\begin{array}{l}\text { No. (\%) with no improvement } \\
\text { (change from baseline } 0 \text { or worst) } \\
\text { from baseline score }\end{array}$} & Baseline & $18(100)$ \\
\hline & 6-month post treatment & $10(56)$ \\
\hline & 12 -month post treatment & $6(17)$ \\
\hline \multicolumn{3}{|l|}{$\begin{array}{l}\text { Investigator's live assessment of severity on a 7-point } \\
\text { ordinal scale }\end{array}$} \\
\hline \multirow[t]{3}{*}{$\begin{array}{l}\text { No. (\%) with at least 2-point improvement from } \\
\text { baseline score }\end{array}$} & Baseline & $0(0)$ \\
\hline & 6-month post treatment & $6(33)$ \\
\hline & 12 -month post treatment & $6(33)$ \\
\hline \multirow[t]{3}{*}{$\begin{array}{l}\text { No. (\%) with at least 1-point improvement from } \\
\text { baseline score }\end{array}$} & Baseline & $0(0)$ \\
\hline & 6-month post treatment & $8(44)$ \\
\hline & 12 -month post treatment & $14(78)$ \\
\hline \multirow{3}{*}{$\begin{array}{l}\text { No. (\%) with no improvement } \\
\text { (change from baseline } 0 \text { or worst) } \\
\text { from baseline score }\end{array}$} & Baseline & $18(100)$ \\
\hline & 6-month post treatment & $10(56)$ \\
\hline & 12-month post treatment & $3(17)$ \\
\hline \multicolumn{3}{|l|}{$\begin{array}{l}\text { Patient's self-assessment of satisfaction with the } \\
\text { change in facial contour defects before and after } \\
\text { treatment on a 5-point ordinal scale } \\
\text { ( } 5 \text { very dissatisfied, } 1 \text { very satisfied) }\end{array}$} \\
\hline \multirow[t]{3}{*}{ Median Score (interquartile range) } & Baseline & $4(1)$ \\
\hline & 6-month post treatment & $2(0)$ \\
\hline & 12 -month post treatment & $2(0)$ \\
\hline \multirow[t]{3}{*}{$\begin{array}{l}\text { No. (\%) with at least 1-point improvement from } \\
\text { baseline score }\end{array}$} & Baseline & $0(0)$ \\
\hline & 6-month post treatment & $18(100)$ \\
\hline & 12-month post treatment & $18(100)$ \\
\hline
\end{tabular}


Table 3. Mean Response score (change from baseline) and Cumulative OR (95\% CI) predicting cumulative probability of Response to therapy.

\begin{tabular}{|c|c|c|c|c|c|}
\hline Variables & $\mathrm{N}$ & $\begin{array}{l}\text { Mean change from } \\
\text { baseline score (SD) }\end{array}$ & $\begin{array}{c}\text { Cumulative } \\
\text { OR }\end{array}$ & $95 \% \mathrm{CI}$ & $P$ value \\
\hline \multicolumn{6}{|l|}{ Age group, years } \\
\hline$<50\left(\operatorname{ref}^{*}\right)$ & 7 & $1.38(0.81)$ & 1 & - & \\
\hline $50-59$ & 7 & $1.53(0.84)$ & 1.82 & $(0.62,5.38)$ & 0.277 \\
\hline$\geq 60$ & 4 & $1.17(0.89)$ & 0.82 & $(0.18,3.78)$ & 0.801 \\
\hline \multicolumn{6}{|l|}{ Body weight, $\mathrm{kg}$} \\
\hline$<50 \mathrm{Kg}\left(\operatorname{ref}^{*}\right)$ & 4 & $1.45(0.77)$ & 1 & - & \\
\hline$>50 \mathrm{Kg}$ & 14 & $1.38(0.87)$ & 0.33 & $(0.077,1.42)$ & 0.137 \\
\hline \multicolumn{6}{|l|}{ Baseline score } \\
\hline $4\left(\operatorname{ref}^{*}\right)$ & 7 & $1.10(0.70)$ & 1 & - & \\
\hline 5 & 10 & $1.57(0.86)$ & 3.35 & $(1.75,6.43)$ & $<0.001$ \\
\hline 6 & 1 & $1.55(1.06)$ & 5.26 & $(0.74,37.13)$ & 0.096 \\
\hline \multicolumn{6}{|l|}{ Cell dose } \\
\hline $1 \mathrm{~mL}\left(\mathrm{ref}^{\star}\right)$ & 11 & $1.30(0.80)$ & 1 & - & \\
\hline $4 \mathrm{~mL}$ & 7 & $1.52(0.91)$ & 1.02 & $(0.46,2.27)$ & 0.958 \\
\hline \multicolumn{6}{|c|}{ Duration post treatment } \\
\hline 6 months $\left(\right.$ ref $\left.^{*}\right)$ & 18 & $1.16(0.81)$ & 1 & - & \\
\hline 12 months & 18 & $1.64(0.84)$ & 3.27 & $(1.86,5.70)$ & $<0.001$ \\
\hline
\end{tabular}

${ }^{*}$ ref: Reference category in regression analysis.

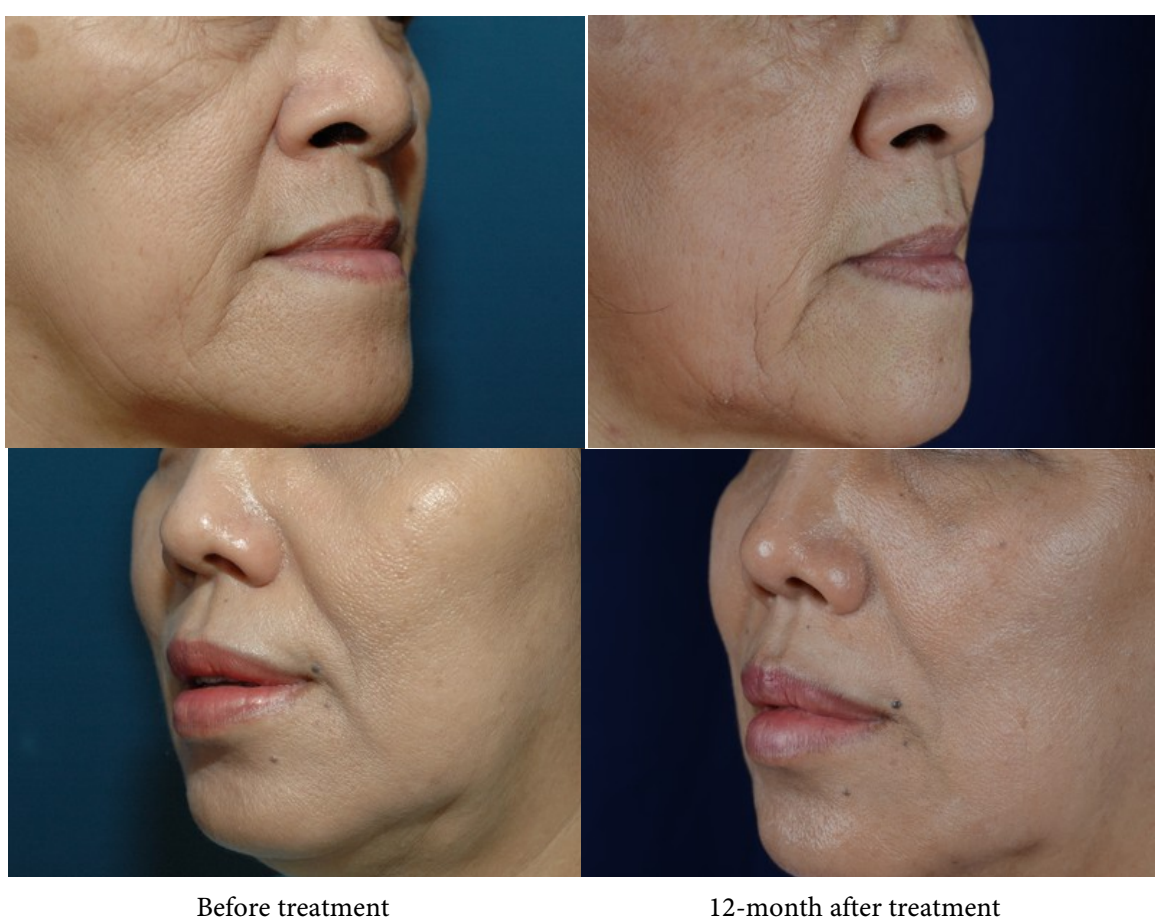

Figure 1. Improvement in nasolabial fold after 3 injections of autologous cultured Fibroblast cells 4 weeks apart at 12-month follow-up. 
marketed as Azficel-T (Laviv ${ }^{\oplus}$ ) by Fibrocell Technologies Inc in the US. In the pivotal study on Azficel-T for variety of facial contour deformities including nasolabial folds [13], $42 \%$ of patients with nasolabial folds had improved by 2-point on a standardized 7-point photoguide as evaluated by the investigators. The corresponding result in our study using the same 7 -point scale is $33 \%$. This could be due to subtle differences in cell culture method or intradermal injection technique between the 2 trials. The most likely explanation though is that the Azficel-T study had required 3 repeated skin biopsies and cell cultures from the freshly biopsied skin prior to each of the 3 injections while we had only performed a single skin biopsy from which fibroblasts are isolated and repeatedly passage to yield all the cells used for all 3 injections.

In this study, we have further investigated the effect of patients' age, duration after treatment (6 and 12 months) and cell dose on the response to ACF treatment. Older patients (age $>60$ years) did show a trend towards poorer response to treatment but this was not statistically significant. The longer the post-treatment follow-up, the better was the response to ACF. This is consistent with ACF being a natural cell based treatment which is slower in producing a noticeable effect compared with synthetic fillers but is capable of producing ongoing and sustained improvement. Interestingly a much larger cell dose (92 million cells) has little additional effect suggesting that the $1 \mathrm{~mL}$ cell dose ( 23 million cells) is near the optimal cell dosing. This was also the cell dose used in the Azficel-T trial [13].

\section{Conclusion}

In conclusion, we have replicated the laboratory and clinical procedures to perform ACF treatment in Malaysia. Our ACF was effective in improving the appearance of nasolabial folds in $22 \%$ of patients, and likely other facial contour defects too.

\section{Acknowledgements}

The authors thank Beijing Cell Technology Inc. for providing the cultured fibroblast cells for this research.

\section{References}

[1] Baumann, L. (2004) Replacing Dermal Constituents Lost through Aging with Dermal Fillers. Seminars in Cutaneous Medicine and Surgery, 2004, 160-167. https://doi.org/10.1016/j.sder.2004.06.006

[2] Coleman, S.R. (2006) Structural Fat Grafting: More Than a Permanent Filler. Plastic and Reconstructive Surgery, 118, 108S-120S. https://doi.org/10.1097/01.prs.0000234610.81672.e7

[3] Yoshimura, K., Sato, K., Aoi, N., Kurita, M., Hirohi, T. and Harii, K. (2008) Cell-Assisted Lipotransfer for Cosmetic Breast Augmentation: Supportive Use of Adipose-Derived Stem/Stromal Cells. Aesthetic Plastic Surgery, 32, 48-55. https://doi.org/10.1007/s00266-007-9019-4 
[4] Sinna, R., Delay, E., Garson, S., Delaporte, T. and Toussoun, G. (2010 )Breast Fat Grafting (Lipomodelling) after Extended Latissimus Dorsiflap Breast Reconstruction: A Preliminary Report of 200 Consecutive Cases. Journal of Plastic, Reconstructive \& Aesthetic Surgery, 63, 1769-1777.

[5] Nishimura, T., Hashimoto, H., Nakanishi, I. and Furukawa, M. (2000) Microvascular Angiogenesis and Apoptosis in the Survival of Free Fat Grafts. Laryngoscope, 110, 1333-1338. https://doi.org/10.1097/00005537-200008000-00021

[6] Yoshimura, K., Sato, K., Aoi, N., et al. (2008) Cell-Assisted Lipotransfer for Facial Lipoatrophy: Efficacy of Clinical Use of Adipose-Derived Stem Cells. Dermatologic Surgery, 34, 1178-1785. https://doi.org/10.1097/00042728-200809000-00003

[7] Coleman, S.R. and Saboeiro, A.P. (2007) Fat Grafting to the Breast Revisited: Safety and Efficacy. Plastic and Reconstructive Surgery, 119, 775-785. https://doi.org/10.1097/01.prs.0000252001.59162.c9

[8] Yoshimura, K., Asano, Y., Aoi, N., et al. (2010) Progenitor-Enriched Adipose Tissue Transplantation as Rescue for Breast Implant Complications. The Breast Journal, 16, 169-175. https://doi.org/10.1111/j.1524-4741.2009.00873.x

[9] TrojahnKolle, S.-F., Fischer-Nielsen, A., Mathiasen, A.B., et al. (2013) Enrichment of Autologous Fat Grafts with ex vivo Expanded Adipose Tissue-Derived Stem Cells for Graft Survival: A Randomised Placebo-Controlled Trial. Lancet, 382, 1113-1120. https://doi.org/10.1016/S0140-6736(13)61410-5

[10] Boss, W.K., Usal, H., Chernoff, G., Keller, G.S., Lask, G.P. and Fodor, P.B. (2000) Autologous Cultured Fibroblasts as Cellular Therapy in Plastic Surgery. Clinics in Plastic Surgery, 27, 613-626.

[11] Watson, D., Keller, G.S., Lacombe, V., Fodor, P.B., Rawnsley, J. and Lask, G.P. (1999) Autologous Fibroblasts for Treatment of Facial Rhytids and Dermal Depressions: A Pilot Study. Archives of Facial Plastic Surgery, 1, 165-170. https://doi.org/10.1001/archfaci.1.3.165

[12] West, T.B. and Alster, T.S. (1998) Autologous Human Collagen and Dermal Fibroblasts for Soft Tissue Augmentation. Dermatologic Surgery, 24, 510-512. https://doi.org/10.1111/j.1524-4725.1998.tb04198.x

[13] Weiss, R., Weiss, M., Beasley, K. and Munavalli, G. (2007) Autologous Cultured Fibroblast Injection for Facial Contour Deformities: A Prospective, Placebo-Controlled, Phase III Clinical Trial. Dermatologic Surgery, 33, 263-268.

[14] Patrikoski, M., Juntunen, M., Boucher, S., et al. (2013) Development of Fully Defined Xeno-Free Culture System for the Preparation and Propagation of Cell Therapy-Compliant Human Adipose Stem Cells. Stem Cell Research \& Therapy, 4, 27. https://doi.org/10.1186/scrt175

[15] Lepperdinger, G., Brunauer, R., Jamnig, A., Laschober, G. and Kassem, M. (2008) Controversial Issue: Is It Safe to Employ Mesenchymal Stem Cells in Cell-Based Therapies? Experimental Gerontology, 43, 1018-1023.

[16] Simon, R. (1989) Optimal Two-Stage Designs for Phase II Clinical Trials. Controlled Clinical Trials, 10, 1-10.

[17] McCullagh, P. (1980) Regression Models for Ordinal Data (with Discussion). Journal of the Royal Statistical Society: Series B, 42, 109-142.

[18] Scott, S.C., Goldberg, M.S. and Mayo, N.E. (1997) Statistical Assessment of Ordinal Outcomes in Comparative Studies. Clinical Epidemiology, 50, 45-55.

[19] Brazer, S.R., Pancotto, F.S., Long, T.T., Harrell, F.E., et al. (1991) Using Ordinal Lo- 
gistic Regression to Estimate the Likelihood of Colo-Rectal Neoplasia. Journal of Clinical Epidemiology, 44, 1263-1270.

[20] Huber, P. (1967) The Behaviour of Maximum Likelihood Estimates under Non-Standard Conditions. Proceedings of the 5 th Berkeley Symposium on Mathematical Statistics and Probability, 1, 221-233.

[21] US Food and Drug Administration (FDA) Approved Products-Autologous Fibroblasts.

http://www.fda.gov/BiologicsBloodVaccines/CellularGeneTherapyProducts/Approv edProducts/ucm260485.htm 